\title{
Advantage of make-to-stock strategy based on linear mixed-effect model: a comparison with regression, autoregressive, times series, and exponential smoothing models
}

\author{
Yu-Pin Liao ${ }^{1}$ and Shin-Kuan Chiu ${ }^{2, *}$ \\ ${ }^{1}$ Chairman's office, Winstar Display Corp., Taichung, Taiwan \\ Ph.D., Program of Business, Feng Chia University, Taichung, Taiwan \\ E-mail: 〈venson@winstar.com.tw〉 \\ ${ }^{2}$ Department of International Trade, Feng Chia University, Taichung, Taiwan \\ E-mail: 〈skchiu@fcu.edu.tw〉
}

\begin{abstract}
In the past few decades, demand forecasting has become relatively difficult due to rapid changes in the global environment. This research illustrates the use of the make-to-stock (MTS) production strategy in order to explain how forecasting plays an essential role in business management. The linear mixed-effect (LME) model has been extensively developed and is widely applied in various fields. However, no study has used the LME model for business forecasting. We suggest that the LME model be used as a tool for prediction and to overcome environment complexity. The data analysis is based on real data in an international display company, where the company needs accurate demand forecasting before adopting a MTS strategy. The forecasting result from the LME model is compared to the commonly used approaches, including the regression model, autoregressive model, times series model, and exponential smoothing model, with the results revealing that prediction performance provided by the LME model is more stable than using the other methods. Furthermore, product types in the data are regarded as a random effect in the LME model, hence demands of all types can be predicted simultaneously using a single LME model. However, some approaches require splitting the data into different type categories, and then predicting the type demand by establishing a model for each type. This feature also demonstrates the practicability of the LME model in real business operations.
\end{abstract}

Keywords: make-to-stock, production strategy, forecasting, linear mixed-effect model, order demand

Received: May 09, 2016; accepted: April 21, 2017; available online: November 30, 2017

DOI: $10.17535 /$ crorr.2017.0023

${ }^{*}$ Corresponding author. 


\section{Introduction}

Nowadays, companies provide a large variety of product to satisfy diversified customer needs. These companies receive customer orders for various types of products, where each order is low volume. This leads to production in the manufacturing process featuring high variations and low volumes (high-mix low-volume). Consequently, the order commitment task becomes more challenging, such as demands for shortened production lead times, diverse customer requirements and more frequent customer orders. Historically, companies usually have a small proportion of orders with high-mix low-volumes. Therefore, most of manufactories that receive highmix low-volume orders usually adopt a make-to-order (MTO) production strategy. Adopting a MTO strategy means defining production schedules immediately after customers have placed an order, which is appropriate for high-mix low-volume orders as such orders include large product variety and a large unpredictable demand fluctuation, where immediately undertaking all production steps immediately after the placement of orders means manufacturers avoid risks. However, when a company receives an increasing number of high-mix low-volume orders, which even becomes the main business model of the company, adopting the MTO strategy gives rise to numerous business operation issues, such as long production lead times, frequently changing production lines, high operating costs and low product quality. All this results in the company developing a usually inefficient complex operation model. Therefore, if issues emerging from high-mix low-volume orders are not resolved, despite growing business revenue, operation costs increase rapidly, product quality falls, and internal employee and customer satisfaction decreases, resulting in stagnant business development. A solution to the problems arising from the MTO strategy for high-mix low-volume orders is to modify the production strategy. What is evident is that companies have in time changed production strategies from the MTO to make-to-stock (MTS), meaning that factories are less inclined to completely adopt an MTO production strategy $[2,52]$. A company adopting the MTS strategy manufactures products and stores them in inventory before customers place orders, and then ship the stored products once the order is placed. The MTS strategy avoids the above-mentioned problems stemming from the MTO strategy. However, the MTS strategy importantly relies on forecasting customer demand which mainly depends on information and reliable forecasting methods. In recent years, demand forecasting has become increasingly complex, primarily due to a changing global economic environment. The underlying reasons for this change can be explained in terms of the following four dimensions: volatility, uncertainty, complexity, and ambiguity (VUCA) [26, 37, 47]. Volatility means that new products are rapidly developed, product lifecycles become shorter, customer preferences quickly change, and organizations are frequently restructured. Consequently, the value of historical data diminishes. Uncertainty stems from unknown factors that cause sudden shifts in demand, and these factors are generally regarded as outliers or interferences. Complexity means that the interaction of these influential factors cannot be modelled easily, whereas ambiguity refers to fuzzy events and situations that cannot be quantifiably defined, leading to a loss of key influential factors. These four dimensions have been shown to influence demand forecasting [8]. In summary, in line with 
the impact of economics on demand forecasting, developing a reliable forecasting method requires analyzing whether historical data can contribute to demand forecasting, and whether the effects of influential factors are identifiable. To meet the requirements of modern forecasting methodologies, this study proposes using the linear mixed-effect (LME) model for forecasting. The LME model has been extensively developed and widely applied in various fields. However, no study has used the LME model to do forecasting in business operations. The LME model is characterized by the inclusion of temporal factors and explanatory variables as well an analysis of their respective significance. Accordingly, crucial influential factors can be identified for the purpose of forecasting demand. These characteristics fulfill the requirements of modern forecasting methodologies and can be used as the basis by companies to improve their operation efficiency and develop competitive advantages. The following sections explore the influence of the MTO and MTS production strategies on business operation as well as the role of forecasting in the MTS strategy, providing a overview of the literature on forecasting methodologies, and summarizing the strengths and weaknesses of commonly used forecasting methods. In addition, the proposed LME model and a method for model parameter estimation are introduced. Subsequently, the order demand for a manufacturer located in Taiwan is forecasted using product type as a crucial explanatory variable. Specifically, the LME model is applied to forecast the order demand for 20 individual product types. A one-year forecast of monthly demand is reported, and three accuracy indices are used to assess the forecasting ability of the model. The results show that the forecasting ability of the LME model in empirical analyses is more stable than that of a regression model, autoregressive model, exponential smoothing model, or a time-series model.

\section{Literature review}

\subsection{Influences of the MTO and MTS on business operations}

Modern production patterns primarily involve two main production strategies: the MTO (based on customer orders) strategy, and the MTS (based on production capacity) strategy [48]. From a customer perspective, one of the competitive advantages of the MTS strategy is the short delivery time and quick response [44]. Therefore, identifying the types of products that are specifically suitable for the MTS strategy or both the MTS and MTO strategies is a favored research topic in management science [48].

Regarding the influence of the MTO and MTS strategies on business operations, Hendry and Kingsman [29] showed that the MTS and MTO strategies are mostly used for manufacturing standard and customized products, respectively. Regarding the attributes of orders, order demand for MTS products is generally predictable, whereas for MTO products it is irregular and unpredictable. Concerning production planning, MTS strategy lines operate according to forecast results, and the production line schedule can be adjusted easily. However, the schedule of MTO strategy lines is determined based on recent order demand, whereas long-term manufacturing schedules are difficult to determine. In terms of product delivery, enterprises that adopt the MTS strategy can ensure rapid product delivery, thus maintain- 
ing high customer satisfaction. The MTO strategy requires long delivery times, and enterprises adopting this strategy must communicate with customers to achieve consensus regarding product delivery time. Concerning product price, compared with the prices of products produced adopting the MTO strategy, the prices of MTS-produced products are relatively more stable. Soman, van Donk, and Gaalman [48] indicated that the MTO strategy is effective for handling orders requesting high-mix customized products; production planning for the MTO strategy must prioritize meeting order demands, while production effectiveness is determined according to crucial elements in the orders (e.g., the expected delivery volume and number of delayed delivery days). The goal of a company that manufactures MTO products is to shorten product delivery times; production efficiency emphasizes the importance of capability planning, orders that are lost due to problems with manufacturing processes, and on-time product delivery. By contrast, the MTS strategy is effective for handling uniform product specifications and less customized products, where production planning is determined based on product demand forecasting and production effectiveness is production-oriented. Therefore, the goal of a company manufacturing MTS products is to enhance product availability, and its production efficiency emphasizes the importance of inventory policy, goods inventory, one-off or batch production, and accurate demand forecasting. Rajagopalan [45] indicated that inventory costs are slightly higher for the MTS strategy than for the MTO strategy, particularly for one-off and batch production. In summary, the MTS strategy relies heavily on the accuracy of product demand forecasting. The advantages of the MTS strategy, including short delivery times, manageable long-term manufacturing schedules, and stable product prices, is realized due to accurate forecasting. In addition, accurate forecasting optimizes inventory levels; therefore, companies applying the MTS strategy control inventory costs in an effective manner. Some researchers have explored the inventory policies and material control mechanisms in the MTO strategy [16]. The forecasting method proposed in this study provides a relatively accurate basis for forecasting random customer orders (demand) for the MTS strategy.

\subsection{Forecasting methodology}

Two main types of forecasting methodologies exist: (1) statistical methods; and (2) data mining and machine learning [12]. Both types of forecasting methodologies are aimed at identifying the relationship between influential factors (independent variables) and research variables (dependent variables), and identifying the effects of the influential factors on research variables [8]. These two methodologies involve distinct approaches to interpreting analysis models. The statistical methodol- ogy is based on data derived from a specific mathematical model as well as unobservable errors. The machine-learning methodology avoids fitting data to a specific model and develops algorithms that are suitable for various types of data. These two methodologies differ in their strengths and characteristics [12]. The statistical methodology uses the probability distribution of errors to infer the significance of the influential factors in a model. The reliability of inferences correlates positively with the mathematical model. The machine learning methodology uses the size of forecast errors as a basis 


\begin{tabular}{|c|c|c|c|}
\hline $\begin{array}{c}\text { Forecasting } \\
\text { method }\end{array}$ & $\begin{array}{c}\text { Can handle } \\
\text { time-correlated } \\
\text { data }\end{array}$ & $\begin{array}{c}\text { Can include } \\
\text { influential } \\
\text { factors }\end{array}$ & $\begin{array}{c}\text { Analyzing the importance } \\
\text { of influential factors } \\
\text { (e.g., } P \text {-value) }\end{array}$ \\
\hline $\begin{array}{c}\text { Linear mixed- } \\
\text { effect model }\end{array}$ & $\bigcirc$ & $\bigcirc$ & $\bigcirc$ \\
\hline $\begin{array}{c}\text { Exponential } \\
\text { smoothing model }\end{array}$ & $\bigcirc$ & $\triangle$ & $\triangle$ \\
\hline ARIMA & $\bigcirc$ & $\triangle$ & $\triangle$ \\
\hline Regression model & $\triangle$ & $\bigcirc$ & $\bigcirc$ \\
\hline
\end{tabular}

Table 1: Characteristics of frequently-used forecasting methods. ( $\bigcirc$ : yea; $\triangle$ : yes following modification by other studies)

for selecting the optimal forecasting model. Several typical forecasting methods are introduced here with the characteristics shown in Table 1. An exponential smoothing model was proposed by Holt [32] and the statistical theoretical foundation for this model was established by Muth [40]. This method involves using a demand observation and predictive value in the current period to determine the predictive value for the subsequent period by using weighted mean. The methodology for exponential smoothing has been developed in recent years to incorporate the effect of influential factors on the accuracy of demand forecasts $[8,24,50]$. Wang [50] used a model selection method where crucial influential factors were included in the selected model, and nonsignificant factors were removed to avoid over-fitting the model.

The time-series model was first developed in the nineteenth century, and past studies on the model were then systematically compiled by Box and Jenkins [10] into a book. A time-series autoregressive integrated moving average (ARIMA) model integrates an autoregressive process and moving average process after obtaining a finite difference from time-series data. The ARIMA model is used to estimate the correlation parameter between the time points of observed values, and the estimated parameter values can then be used for forecasting. Subsequently, Box and Tiao [11] added other time-series influential factors to the ARIMA model. Pankratz [42] called this model the dynamic regression model. The regression model is a type of linear model most frequently mentioned in statistical analyses. The regression model assumes that research variables and influential factors are linearly related, and can thus be used to explore the effect of influential factors on research variables. Furthermore, the regression model assumes that observation values are mutually independent; hence, it is applicable for analyzing data containing mutually independent observation values. If the model is used to analyze time-correlated data, i.e., the observation values are correlated over time, then unbiased but invalid model coefficient estimators can be obtained. Consequently, the standard errors of the model coefficient estimators are incorrect, and problems regarding statistical testing within the models arise, such as whether the model coefficients are significantly greater than 0 , whether the models exhibit explanatory power, and whether the predictive intervals are reliable in forecast analysis [27, 18]. The LME model is considered as an extension of the linear model. The LME model adds random effects to the linear model which otherwise only has fixed effects. Hence, a model that has both fixed and random effects is called an LME model. This specific model is typically used to describe the relationship be- 
tween research variables and categorical factors with correlated observation values. A characteristic of the LME model is that observation values at the same categorical level have identical random effect values for de- pendent variables; observation values at different levels have distinct values of a random effect. This characteristic explains the correlation between observation values at an identical level. Therefore, the LME model differs considerably from the regression model. The LME model can be applied to data where observation values are correlated (e.g., longitudinal data, repeated measures data, and multilevel data). However, the regression model can only be applied to data where the observation values are mutually independent. In industrial operations, the pattern of data observations is often time-correlated. For example, when forecasting monthly product demand or monthly inventory levels, the observa- tion values are correlated over time. Under such circumstances, the LME model is more accurate than the regression model for identifying statistically significant factors. The ARIMA model is one of the most often used sales forecasting models for a variety of areas $[23,33]$. Exponential smoothing is a nonparametric method and was found to perform well for outliers [22]. The regression model is one of the main forecasting techniques [28]. These three methods are widely applied in industries that demand forecasting $[15,25,6,7,20]$. However, in the past three years, the LME model has been broadly applied in various fields, such as the timber industry [30], medicine [9, 49], and ecology [31], and to identify crucial influential factors. In addition, numerous studies have established models for forecasting $[38,46]$. In industrial engineering and management science, no study has used the LME model to make predictions using time-correlated data or to identify key influential factors $[1,3,18,21]$. Therefore, in this study, an LME model was applied to business operations to analyze the importance of influential factors, and to forecast product demand. In addition, the performance of the LME model was compared with the regression model, autoregressive model, times series model, and exponential smoothing model, which are the research contributions provided by this study.

\section{Linear mixed-effect (LME) model}

According to parameter attributes, two types of effects exist in a LME model: fixed and random effects [43, 51]. In linear model, the parameters are all fixed values and therefore its corresponding covariates are referred to as fixed-effect parameters. The fixed effect describes the true value of the coefficient for an entire population, or the true value of the coefficient for a factor that can be repeatedly tested under identical conditions. If a factor in a model exhibits a random effect, then the factor is sampled from an entire population. The random effect is a coefficient of the factor; moreover, the coefficient is a random variable and not a fixed value. The following section introduces the LME model developed by Laird and Ware [36], and describes

how the research variables are forecasted. The following is an LME model developed by Laird and Ware [36], and expresses as follows:

$$
\begin{aligned}
\boldsymbol{y}_{i} & =\boldsymbol{X}_{i} \boldsymbol{\beta}+\boldsymbol{Z}_{i} \boldsymbol{b}_{i}+\boldsymbol{\epsilon}_{i}, i=1, \ldots, M \\
\boldsymbol{b}_{i} & \sim N(\mathbf{0}, \boldsymbol{\Psi}), \boldsymbol{\epsilon}_{i} \sim N\left(\mathbf{0}, \boldsymbol{\Lambda}_{i}\right),
\end{aligned}
$$


where $\boldsymbol{b}_{i}$ is a matrix that is independent of $\boldsymbol{\epsilon}_{i}$ (index $i$ denotes the $i$ th group at a single level), $\boldsymbol{y}_{i}$ contains $n_{i}$ observation values for the $i$ th group, $M$ denotes the number of groups, $\boldsymbol{\beta}$ denotes a $p$-dimensional vector for the fixed effect, $\boldsymbol{b}_{i}$ denotes a $q$-dimensional vector for the random effect, $\boldsymbol{X}_{i}$ denotes an $n_{i} \times p$ design matrix for the fixed effect, $\boldsymbol{Z}_{i}$ is an $n_{i} \times q$ design matrix for the random effect, and $\boldsymbol{\epsilon}_{i}$ denotes an $n_{i}$-dimensional within-group random error term. The variable $\boldsymbol{\epsilon}_{i}$ obeys a multivariate normal distribution with an expected value of $\mathbf{0}$ and a covariance matrix of $\boldsymbol{\Lambda}_{i}$, and $\boldsymbol{b}_{i}$ obeys a multivariate normal distribution with an expected value of $\mathbf{0}$ and a covariance matrix of $\boldsymbol{\Psi}$. The model assumes that $\boldsymbol{\epsilon}_{i}$ and $\boldsymbol{\epsilon}_{j}$ are mutually independent $(i \neq j)$; in addition, $\boldsymbol{\epsilon}_{i}$ and $\boldsymbol{b}_{i}$ are mutually independent. Therefore, considering Models (1) and (2), the covariance matrix of the within-group observation values $\boldsymbol{y}_{i}$ is expressed as follows:

$$
\boldsymbol{V}_{i}:=\operatorname{Var}\left(\boldsymbol{y}_{i}\right)=\operatorname{Var}\left(\boldsymbol{Z}_{i} \boldsymbol{b}_{i}\right)+\operatorname{Var}\left(\boldsymbol{\epsilon}_{i}\right)=\boldsymbol{Z}_{i} \boldsymbol{\Psi} \boldsymbol{Z}_{i}^{T}+\boldsymbol{\Lambda}_{i},
$$

where the nondiagonal elements of $\boldsymbol{V}_{i}$ are not required to be 0 . Therefore $\boldsymbol{V}_{i}$, the covariance matrix of $\boldsymbol{y}_{i}$, is derived from (1) and (2), which allows the existence of the correlation between observation values within a group. This is the major difference between LME model and linear model. The feature of the covariance matrix (3) is that observation values of the different groups are independent of each other, and the within-group observation values are intercorrelated. The most often used methods to estimate the parameters in the LME model are the maximum likelihood (ML) and restricted ML (REML) estimation methods. Regarding the ML method, the estimates of ML estimators are those that reach the maximum value of ML functions. The REML estimate of $\boldsymbol{\beta}, \hat{\boldsymbol{\beta}}$, is obtained by applying an iterative method to a restricted natural-logarithm ML function.

$$
\begin{aligned}
l_{\mathrm{REML}}= & -\frac{1}{2}\left(\ln (2 \pi)\left(\sum_{i} n_{i}-p\right)+\sum_{i} \ln \left(\operatorname{det}\left(\boldsymbol{V}_{i}\right)\right)\right. \\
& \left.+\sum_{i}\left(\boldsymbol{y}_{i}-\boldsymbol{X}_{i} \hat{\boldsymbol{\beta}}\right)^{T} \boldsymbol{V}_{i}\left(\boldsymbol{y}_{i}-\boldsymbol{X}_{i} \hat{\boldsymbol{\beta}}\right)+\sum_{i} \ln \left(\operatorname{det}\left(\boldsymbol{X}_{i}^{T} \boldsymbol{V}_{i} \boldsymbol{X}_{i}\right)\right)\right) .
\end{aligned}
$$

By comparison, the REML method is aimed at identifying the estimators that exhibit unbiased characteristics. Therefore, estimators obtained using the REML method from (4) are unbiased, whereas those derived using the ML method could be biased or unbiased. Therefore, most researchers prefer the REML method [43, 51]. We also use the REML method in this study. After the explanatory variables $\boldsymbol{X}_{i}^{\text {new }}$ and

$\boldsymbol{Z}_{i}^{\text {new }}$ have been obtained, the estimates of $\boldsymbol{\beta}$ and $\boldsymbol{b}_{i}$ (i.e., $\hat{\boldsymbol{\beta}}$ and $\hat{\boldsymbol{b}}_{i}$ ) obtained from REML method can be used to forecast the research variable $\boldsymbol{y}_{i}$. The predictive value is as follows:

$$
\hat{\boldsymbol{y}}_{i}=\boldsymbol{X}_{i}^{\text {new }} \hat{\boldsymbol{\beta}}+\boldsymbol{Z}_{i}^{\text {new }} \hat{\boldsymbol{b}}_{i}
$$

\section{A case study}

This study adopted a single-level LME model to forecast product demand. In the case study, the sample was acquired from a leading professional industrial LCD/OLED 


\begin{tabular}{|c|c|c|c|c|}
\hline Year & $\begin{array}{c}\text { Number of } \\
\text { orders }\end{array}$ & $\begin{array}{c}\text { Total product } \\
\text { demand (piece) }\end{array}$ & $\begin{array}{c}\text { Average product demand } \\
\text { (piece) per order }\end{array}$ & $\begin{array}{c}\text { product } \\
\text { types }\end{array}$ \\
\hline 2011 & 12,911 & $3,601,903$ & 278.98 & 2,714 \\
\hline 2012 & 17,961 & $8,342,283$ & 464.47 & 3,514 \\
\hline 2013 & 20,164 & $6,720,794$ & 333.31 & 4,541 \\
\hline 2014 & 22,591 & $8,062,995$ & 356.91 & 5,823 \\
\hline 2015 & 22,362 & $9,045,073$ & 404.48 & 5,467 \\
\hline
\end{tabular}

Table 2: Number of orders and product demand during year 2011 2015

display manufacturer in Taiwan. This manufacturer produces products that are critical components of various devices used in daily life and are applied in various industries. Moreover, the company has an international customer base. Table 2 shows the number of orders, total product demand, average product demand per order, and product types from 2011 to 2015. Until 2015, the manufacturer produced more than 5,000 product types, and the average quantity of products required in an order was approximately 400. Thus, the manufacturer is considered a suitable example of a business that produces a diverse combination of product types, and shows that the manufacturer receives a large number of high-mix low-volume orders.

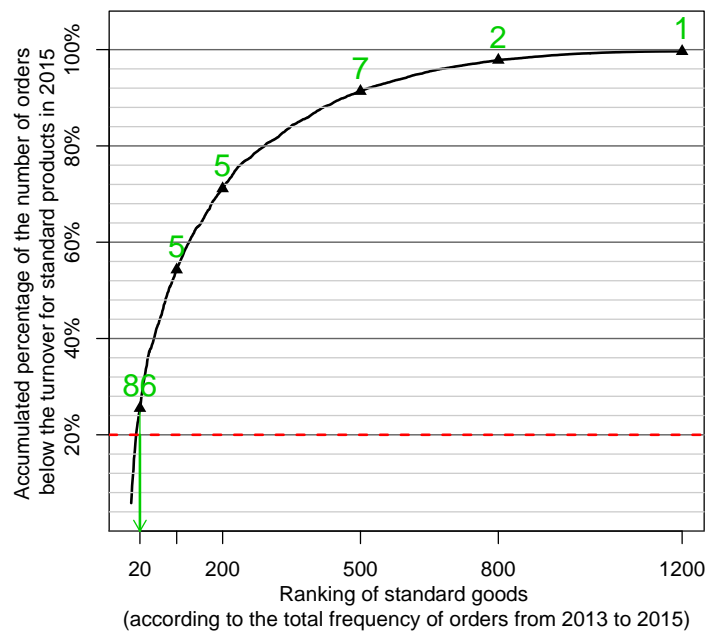

Figure 1: The plot shows that the accumulated percentage of the maximum total number of orders from 2013 to 2015 is less than the turnover of standard products in 2015. The first 20 products accounted for approximately $25 \%$ of the turnover from standard products. The numbers in green denote the number of orders for standard products in 2015 corresponding to the horizontal axis.

A characteristic of high-mix low-volume manufacturers is that they typically commence produc- tion only after receiving a customer order. This production pattern is typical of the MTO strategy, which is adopted to serve customers of this manufacturer. In recent years, the manufacturers profits have decreased despite increasing revenue and market share. Therefore, the manufacturer planned to change its pro- 
duction strategy by adopting the MTS strategy for some product types in order to increase batch production capacity, reduce production costs, and improve production efficiency. In addition, the manufacturer believed that adopting the MTS strategy would enhance customer satisfaction by ensuring rapid lead time of production, thereby providing a competitive advantage. Thus, the ability to accurately forecast product demand is important. This study selected the top 20 standard products most frequently ordered between 2013 and 2015 by customers of the sample manufacturer with the intention of implementing the MTS strategy. As shown in Figure 1, these 20 standard products accounted for $25 \%$ of the manufacturers turnover from standard products in 2015, with 86 orders placed in the same year. After implementing the MTS strategy, the manufacturer planned to run production of each product type once per month. Accordingly, the production frequency, cost of handling orders, and frequency of changing production lines was reduced. Thus, the long-term production capacity plans are implemented to maximize the benefits of producing a high volume of these 20 products with fewer runs.

\subsection{Data structure}

The data structure comprises 20 types of standard products. Data on monthly product demand for each product type were collected in the period from January 2009 to December 2015. Historical data prior to 2014 were used to estimate model parameters, and the model was used to forecast product demand for the period January-December 2015. Not all of the 20 products were manufactured from 2009. The historical data used to estimate model parameters comprises 1295 observation values (64 observation values on average for each product type). The product lifecycle varied by year, and the product demand varied by month. Therefore, year and month were crucial predictors. For each type of product, the monthly product demands in each month were related. In this study, the explanatory variables (year and month) were added to the LME model to analyze the monthly product demand data. Regarding product sales, the product demand varied by product type. Accordingly, product type was regarded as a crucial explanatory variable as it had an influence on forecasting product demand. In this study, based on the characteristics of the LME model, we used product type as a random-effect term. By doing so, we are able to forecast the demand for each product type in one LME model. Unlike the LME model, some of the methods we compared in this paper did not have a universal model to account for the differences between 20 unique product types. Therefore, for these forecasting methods, the data are divided into several data sets based on product type, and a model is constructed for each partitioned data in order to forecast the demand for each product type. This approach substantially reduces the sample size and the forecast accuracy.

\subsection{Model development}

Product demand differed by product type, hence we assumed the demand for each type of product to be mutually independent. In the following model, we consider two main covariates, time and product types, which should correlate with the ten- 
dency for product demand. We separate the time effect into year and month effects. The year effect is used to account for growth of demand and the month effect for the seasonal tendency of demand. Product types are used to account for demand differences in every product type. In Model (1), which is the single-level model, the random effect was set as the product type given that products of different product types have different product demands, which in turn accounts for the effect variation in product types. The model is expressed as follows:

$$
\begin{aligned}
\boldsymbol{y}_{i}= & \beta_{0}+\beta_{1}(\text { year }-2009)+\beta_{2}(\text { year }-2009)^{2}+\text { month } \times \boldsymbol{\beta}_{3}+ \\
& b_{i 0}+b_{i 1}(\text { year }-2009)^{2}+\boldsymbol{\epsilon}_{i}
\end{aligned}
$$

where $\boldsymbol{y}_{i}$ is a vector that denotes the monthly product demand (the vector length is equal to the data quantity for product $i$ ); $\beta_{0}, \beta_{1}, \beta_{2}$, and $\boldsymbol{\beta}_{3}$ denote the intercept, year, year-squared, and month for the fixed-effect term; and $b_{i 0}$ and $b_{i 1}$ denote the intercept and year-squared for the random-effect term. To account for the growth of demand, year was regarded as a continuous variable with 2009 used as the baseline, and a year-squared covariate is included allowing the tendency of demand to be quadratic, not restricted as linear. To account for the seasonal tendency of demand, month was regarded as a nominal categorical variable, which accounts for the busy and slack seasons of product demand. The intercept is set with randomeffect in product types, which gives each product type an intercept value and thus accounts for the average difference in demands between product types. We also set the year-squared covariate in the random-effect as this allows each product type to have a unique quadratic demand tendency, enabling the model to reflect the current situation more accurately. In expressing Equation (1) as Model (6), the fixed-effect explanatory variable $\boldsymbol{X}_{i}$ is a matrix comprising a column of 1's vector for the intercept, year, year-squared, and month covariates. Month was a categorical variable; therefore, the month term in Model (6) was encoded as a dummy variable. The dummy variable for month had 11 indicator variables with a value of 0 or 1 , and the total product demand in January is used as the baseline. Thus, the expression $\boldsymbol{\beta}=\left[\beta_{0}, \beta_{1}, \beta_{2}, \boldsymbol{\beta}_{3}^{T}\right]^{T}$ is a $14 \times 1$ vector, where $\boldsymbol{\beta}_{3}$ is the coefficient of the dummy variable for the month covariate and has 11 elements. The explanatory variable $\boldsymbol{Z}_{i}$ in the random-effect explanatory variable comprised the intercept and year-squared covariate, where the coefficients are a $2 \times 1$ vector expressed as $\boldsymbol{b}_{i}=\left[b_{i 0}, b_{i 1}\right]^{T}$. In Model (6), the year-squared covariate in the random-effect explanatory variable was also a part of the fixed-effect explanatory variable, and was used to account for the fact that the expectation of $\boldsymbol{b}_{i}$ was probably unequal to $\mathbf{0}$; thus, the assumption that $\boldsymbol{b}_{i}$ in (2) was equal to $\mathbf{0}$ was reasonable. To forecast the monthly product demand for 2015, 2015 was used as the value for the year and year-squared covariates. Both covariates and the target month were input into the explanatory variable to form $\boldsymbol{X}_{i}^{\text {new }}$ and $\boldsymbol{Z}_{i}^{\text {new }}$. Subsequently, $\hat{\boldsymbol{\beta}}$ and $\hat{\boldsymbol{b}}_{i}$ in (5) were used to obtain the forecasted value $\hat{\boldsymbol{y}}_{i}$. 


\subsection{Other forecasting methods}

Comparing forecasting methods is crucial in methodological studies [41, 34, 13, 19, 4]. The model proposed in this study was compared with commonly used statistical forecasting methods, and we incorporate the two main covariates, time and product types, into the following models. We begin with the following regression model:

$$
\begin{aligned}
Y_{j}= & \alpha_{0}+\alpha_{1}\left(\text { year }_{j}-2009\right)+\alpha_{2}\left(\text { year }_{j}-2009\right)^{2}+\text { month } \times \boldsymbol{\alpha}_{3} \\
& + \text { productType } \times \boldsymbol{\alpha}_{4}+\left(\text { year }_{j}-2009\right)^{2} \times \text { productType } \times \boldsymbol{\alpha}_{5}+\delta_{j}
\end{aligned}
$$

where $\alpha_{0}, \alpha_{1}, \alpha_{2}, \boldsymbol{\alpha}_{3}, \boldsymbol{\alpha}_{4}$ and $\boldsymbol{\alpha}_{5}$ are regression coefficients and $\boldsymbol{\alpha}_{3}, \boldsymbol{\alpha}_{4}$ and $\boldsymbol{\alpha}_{5}$ denote the coefficients of the dummy variables for the covariates month, productType and the intersection of year square and productType, respectively, and $\delta_{j}$ is the error term. Model (7) contains year and year-squared covariates to account for the annual growth trend, and contains the interaction effect of product type and year square to account for the unique trend for each product type. The product type nominal categorical effect is included to account for the baseline differences between product types. The month nominal categorical effect is included as well, to account for the seasonal tendency of demand. Model (7) (i.e., the regression model) is designed to have as much covariates information as Model (6) (i.e., the LME model). The dummy variable for 20 product types, productType, had 19 indicator variables to summarize the information in product types, which can correspond to the randomeffect intercept term in Model (6). The intersection covariate, $\left(\text { year }_{j}-2009\right)^{2} \times$ productType, is designed to correspond to the year-square random-effect in Model (6). Therefore, Model (7) can be compared with Model (6) to examine differences when some covariates are assumed to be random-effect in Model (6). A total of 1295 observations of monthly product demand $\left(Y_{j}, j=1, \ldots, 1295\right)$ were used to estimate the coefficients in Model (7) and to obtain the significance of the coefficients with $P$ values. In the results section, Models (6) and (7) are compared for forecast accuracy and the $P$-values. We also use the autoregressive model to compare with the LME model. Given that the error term in this model is autoregressively correlated and products in different product types should be independent, we build one product type with one autoregressive model. The data were divided into 20 data sets according to each product type, and yielded an average of 64 samples for each type of product. Hence each product type has its own regression coefficients, and the product type effect does not need to be included in this model. For comparison with Models (6) and (7), we include year, year-squared and month effects into this model. The meaning of these effects are the same as in Models (6) and (7), except that they are built for each product type separately. The autoregressive model is presented as follows:

$$
Y_{j}=\bar{\alpha}_{0}+\bar{\alpha}_{1}\left(\text { year }_{j}-2009\right)+\bar{\alpha}_{2}\left(\text { year }_{j}-2009\right)^{2}+\text { month } \times \overline{\boldsymbol{\alpha}}_{3}+\bar{\delta}_{j},
$$

where $\bar{\delta}_{j}$ is structured as AR1 correlated errors. Model (8) is established for each of the 20 product types and therefore each product type has its own estimated coefficients for intercept, year, year-square and month covariates. Hence, applying Model (8) to each of product type can generate more covariate information than 
that in Models (6) and (7) where the coefficients of year and month covariates are assumed to be the same for all product types. Next, the model proposed in this study was compared with the exponential smoothing model, in which the product demand observation values $Y_{t}$ 's and their predictive values $F_{t}$ 's were used to obtain the predictive values for the subsequent period by calculating a weighted mean. The forecast formula is as follows:

$$
F_{t+1}=\alpha Y_{t}+(1-\alpha) F_{t}
$$

where $\alpha$ is the weighted coefficient. To accurately forecast the monthly product demand in this case, we adjusted the exponential smoothing model to account for two influential factors (i.e., month and product type). The data were divided into 20 data sets according to each product type, and each data set was divided into 12 subsets (one for each month). For each product type, no more than six observations from each month in the historical data were used. The pre-2014 monthly product demand data were used to forecast the product demand for the corresponding months in 2015. The weighed coefficient was $\alpha=\frac{1}{2(N+1)}$, where $N$ is the number of observations for a month $(N \leq 6)$. Finally, the model proposed in this study was compared with a seasonal time-series model; specifically, the autoregressive integrated moving average model (denoted by SARIMA), which was considered to be a suitable model given that the data were not nonstationary time-series data. The mathematical model for $\operatorname{SARIMA}(p, d, q)(P, D, Q)_{s}$ is expressed as follows: $p, d$, and $q$ are the order of autoregressive process, degree of differencing, and the order moving-average process in ARIMA, respectively; $s$ is a seasonal parameter; and $P, D$, and $Q$ refer to the autoregressive, differencing, and moving average terms for the seasonal part of the ARIMA model:

$$
\begin{gathered}
\left(1-\sum_{i=1}^{p} \phi_{i} B^{i}\right)\left(1-\sum_{i=1}^{P} \Phi_{i} B^{s \times i}\right)(1-B)^{d}\left(1-B^{s}\right)^{D} Y_{t} \\
=\left(1+\sum_{i=1}^{q} \theta_{i} B^{i}\right)\left(1+\sum_{i=1}^{Q} \Theta_{i} B^{s \times i}\right) \xi_{t}
\end{gathered}
$$

where $\phi_{i}$ is the $i$ th order autoregressive process coefficient, $\theta_{i}$ is the $i$ th order movingaverage process coefficient, $d$ is degree of nonseasonal differencing, $\Phi_{i}$ is the $i$ th order seasonal autoregressive process coefficient, $\Theta_{i}$ is the $i$ th order seasonal movingaverage process coefficient, $D$ is the degree of seasonal differencing, $B$ is a backward shift operator, and $\xi_{t}$ is a normally distributed confounding term. Longitudinal data were collected for each of the 20 product types. A time-series model was established for each of the 20 product types. In this case, the month was regarded as a crucial influential factor for forecasting, and thus the seasonal parameter $s$ was set to 12 , which indicates the existence of correlations in the data for every 12 month. The samples were categorized by product type, yielding an average of 64 samples for each type of product. The parameters in Model (9) were determined based on the characteristics of an autocorrelation function, a partial autocorrelation function, and an extended autocorrelation function. Finally, the $\operatorname{SARIMA}(2,0,2)(1,0,1)_{12}$ model was used to forecast the product demand for each product type. 


\subsection{Results}

In this study, the mean of absolute error (MAE), mean of absolute percent error (MAPE), and root-mean-square error (RMSE) were used as accuracy indices. The definitions for these accuracy indices are provided as follows:

$$
\begin{aligned}
\text { MAE } & =\frac{1}{n} \sum_{t=1}^{n}\left|F_{t}-Y_{t}\right| \\
\text { MAPE } & =\frac{100}{n} \sum_{t=1}^{n}\left|\frac{F_{t}-Y_{t}}{Y_{t}}\right| \\
\text { RMSE } & =\left(\frac{1}{n} \sum_{t=1}^{n}\left(F_{t}-Y_{t}\right)^{2}\right)^{0.5}
\end{aligned}
$$

where $n$ denotes the number of months to be forecasted ( $n=12$ in this case), $Y_{t}$ represents the true product demand for month $t$ of 2015 , and $F_{t}$ is the forecasted product demand for month $t$. The LME model was compared with that in the regression model. Since the random-effect terms in the LME model may correspond to the intersection effects in the regression model, we only compare the other fixedeffects appearing in both the LME model and the regression model (Table 3).

\begin{tabular}{|c|c|c|l|c|c|l|}
\hline & \multicolumn{3}{|c|}{ Linear mixed-effect model } & \multicolumn{3}{|c|}{ Regression model } \\
\hline $\begin{array}{c}\text { Explanatory } \\
\text { variable }\end{array}$ & Cf. & S.e. & $P$-value & Cf. & S. e. & $P$-value \\
\hline The intercept term & 39.46 & 320.14 & .9019 & -544.04 & 439.33 & .2158 \\
\hline (Year-2009) & 800.55 & 133.52 & $.0000^{* * *}$ & 828.02 & 134.28 & $.0000^{* * *}$ \\
\hline (Year-2009) $)^{2}$ & -99.97 & 25.82 & $.0001^{* * *}$ & -102.42 & 36.11 & $.0046^{* *}$ \\
\hline February & 206.41 & 283.03 & .4660 & 214.72 & 283.04 & .4482 \\
\hline March & 736.88 & 281.57 & $.0090^{* *}$ & 742.45 & 281.56 & $.0085^{* *}$ \\
\hline April & 753.30 & 281.56 & $.0076^{* *}$ & 755.41 & 281.55 & $.0074^{* *}$ \\
\hline May & 536.51 & 280.89 & $.0564-$ & 547.05 & 280.90 & $.0517-$ \\
\hline June & 253.56 & 281.62 & .3681 & 266.27 & 281.63 & .3446 \\
\hline July & 591.73 & 271.46 & $.0295^{*}$ & 605.51 & 271.49 & $.0259 *$ \\
\hline August & 91.35 & 271.48 & .7366 & 105.46 & 271.53 & .6978 \\
\hline September & 711.75 & 271.46 & $.0088^{* *}$ & 727.24 & 271.49 & $.0075{ }^{* *}$ \\
\hline October & 297.69 & 271.05 & .2723 & 313.23 & 271.09 & .2481 \\
\hline November & 473.91 & 272.52 & $.0823-$ & 489.27 & 272.56 & $.0729-$ \\
\hline December & 360.30 & 270.62 & .1833 & 380.94 & 270.74 & .1597 \\
\hline
\end{tabular}

Cf. $=$ Coefficient, S. e. $=$ Standard error

Table 3: The linear mixed-effect model versus the regression model. ("-": P-value $\leq .1$; “*”: $P$-value $\leq .05$; “**": P-value $\leq .01 ;$ “***": P-value $\leq .001)$

As shown in Table 3, the absolute values of the coefficients for the common effects (not including intercept term) in the LME model are smaller (i.e., close to 0) than all of those in the regression model. In addition, the $P$-values for all of the common effects in the LME model are greater than those in the regression model except for the year-square term, and the standard errors for all of the common effects in 
the LME model are smaller than the regression model except for the March and April terms. However, the regression model is suitable for data containing mutually independent observation values. In this case, the observation values for product demand were correlated over time, thereby violating the assumption of the regression model. Therefore, the standard errors and $P$-values for the regression model (Table 3 ) are not valid estimates [27, 18], whereas those for the LME model are more reliable.

\begin{tabular}{|c|c|c|c|c|c|c|}
\hline & \multicolumn{2}{|c|}{ MAE } & \multicolumn{2}{c|}{ MAPE } & \multicolumn{2}{c|}{ RMSE } \\
\hline & Mean & SD & Mean & SD & Mean & SD \\
\hline Linear mixed-effect model & 1412.71 & 1500.04 & $152 \%$ & $150 \%$ & 1849.42 & 1919.86 \\
\hline Regression model & 1421.39 & 1397.85 & $191 \%$ & $286 \%$ & 1845.34 & 1805.35 \\
\hline Autoregressive model & 1568.93 & 1569.31 & $242 \%$ & $286 \%$ & 1965.46 & 2123.27 \\
\hline SARIMA(2,0,2)(1,0,1) 12 & 1385.85 & 1697.60 & $212 \%$ & $239 \%$ & 1801.69 & 2177.72 \\
\hline Exponential smoothing model & 1565.54 & 1547.88 & $201 \%$ & $177 \%$ & 2003.87 & 2193.16 \\
\hline
\end{tabular}

Table 4: Accuracy indices for the five forecasting models

Table 4 shows the accuracy indices for the five forecasting methods. Given that this case involved three accuracy indices for each of the 20 product types, Table 4 presents the mean and standard deviation (SD) of the three accuracy indices over the 20 products. As shown in Table 4, the mean values of MAE, MAPE, and RMSE for the LME model and the regression model are smaller than those for the exponential smoothing model, and the autoregressive model. The SD values for the LME model and the regression model are smaller than those of the other three models, except that the SD value of MAPE for the regression model is the largest value among the models. Although $\operatorname{SARIMA}(2,0,2)(1,0,1)_{12}$ has the smallest mean values in MAE and RMSE, it also has larger SD values. This result indicates that, in this case, the performance of the LME model is generally more stable than the other four methods.

The model comparison is shown in Table 5. Since product type and month effects are regarded as crucial influential factors in this empirical study, we have to consider these effects into models. For the models that cannot add these effects directly, we divided dataset into several parts so that the forecasts will depend on these two factors. Then for each product type, the pre-2014 monthly data were used to forecast the monthly product demand for 2015. In this manner, the exponential smoothing model need divide data into 20 data sets according to each product type, and each data set was divided into 12 subsets for each month, and applied exponential smoothing model to the 240 data sets, and therefore used 240 models. Hense, less than six observations from the historical data were used in the exponential smoothing model (for a given month, there were at most 6 sets of data from 2009 to 2014); consequently, the risk of inferential error was high because only a few observations were involved in the prediction. Regarding the seasonal time-series model SARIMA $(2,0,2)(1,0,1)_{12}$, since the month effect can be added into the seasonal parameter, we divided the data into 20 data sets according to product type for adding the product effect. For each product type, 64 observations were used on average. The SARIMA $(2,0,2)(1,0,1)_{12}$ model was used to forecast the product demand for each product type by considering the correlation between the data for every 12 
month. Since the autoregressive model can add the month effect into covariates, it applies the 20 data sets divided by the product type to the autoregressive model for jointing the product type effect. Accordingly, although such a procedure may take into account the various monthly effects for various product types and the interaction between product type and month, it reduces the number of data observations in the prediction. In the LME model and the regression model, product type and month effects are incorporated into covariate variables, and 1295 data observations were used to estimate the coefficients in the models. The number of data observations used in both models was considerably more than that used in the autoregressive model, the exponential smoothing and the time-series models, which might explain why the LME model and the regression model produced lower forecast errors. Furthermore, in the LME model, we took into consideration the random effects for all effects (including month and year terms) and applied the likelihood ratio test to examine whether these terms are significant for inclusion into Model (6). The results showed that only the random effects of the intercept and year-squared terms were significant, and the random effects of the month and year terms did not significantly increase the explanatory power of the LME model. Therefore, the random effect of the month and year terms were not included in Model (6). This testing result is also due to the fact that we did not include the intersection effect between the product type and both the month and year terms in Model (7). Therefore, Model (7) can be compared with Model (6) under a similar covariate information condition.

\begin{tabular}{|c|c|c|c|c|c|}
\hline & $\begin{array}{c}\text { Number of } \\
\text { models }\end{array}$ & $\begin{array}{c}\text { Number of } \\
\text { samples }\end{array}$ & $\begin{array}{c}\text { The effect of } \\
\text { product type }\end{array}$ & $\begin{array}{c}\text { The effect } \\
\text { of month }\end{array}$ & $\begin{array}{c}\text { The effect of } \\
\text { interaction }\end{array}$ \\
\hline $\begin{array}{c}\text { Linear mixed- } \\
\text { effect model }\end{array}$ & 1 & 1,295 & $\bigcirc$ & $\bigcirc$ & $\triangle$ \\
\hline $\begin{array}{c}\text { Regression } \\
\text { model }\end{array}$ & 1 & 1,295 & $\bigcirc$ & $\bigcirc$ & $\triangle$ \\
\hline $\begin{array}{c}\text { Autoregressive } \\
\text { model }\end{array}$ & 20 & $\leq 72$ & $\bigcirc$ & $\bigcirc$ & $\bigcirc$ \\
\hline $\begin{array}{c}\text { SARIMA } \\
(2,0,2)(1,0,1)_{12}\end{array}$ & 20 & $\leq 72$ & $\bigcirc$ & $\bigcirc$ & $\bigcirc$ \\
\hline $\begin{array}{c}\text { Exponential } \\
\text { smoothing model }\end{array}$ & 240 & $\leq 6$ & $\bigcirc$ & $\bigcirc$ & $\bigcirc$ \\
\hline
\end{tabular}

Table 5: Comparison of the five models. The interaction represents the interaction term between product type and month. ( $\bigcirc$ : under consideration; $\triangle$ : removed because of nonsignificance)

\section{Discussion and conclusion}

In this paper, we propose using the LME model as a vehicle for forecasting in business operations. In summary, when applying the LME model, all of the historical data were used in one model to predict the monthly product demand for each product type, and to avoid problems associated with dividing data into smaller data sets. In this case study, the LME model enables manufacturers who adopt the MTS strategy to predict the amount of required inventory. Furthermore, the model is more stable for forecasting product demand than the regression, autoregressive 
time-series, and exponential smoothing models. Similar to the linear model, the LME model is typically used to examine the relationship between explanatory and research variables. Unlike the linear model, which assumes the observation values to be mutually independent, the LME model is suitable for examining correlated data. Because the data pertaining to business operations are generally correlated over time, whereas the linear model is limited in its applicability. Regarding the linear model with specific autocorrelated errors, there is a difficultly in using existing statistical software to arbitrary model the structure of an error covariance matrix that matches the rationality of real data. Take the data studied in this paper as an illustration. The observation values within the same product type can be modeled as AR1 error structure and the values between product type should be modeled as independent. This type of autocorrelative error structure is difficult to treat in a linear model, even when using the existing popular statistical software. This is the main reason why we divide the entire data into 20 data sets and fit the AR1 error model on each data set because, by doing so, the problem where observation values between product types are to be modeled as independent is addressed. By contrast, the LME model was initially developed to handle correlated data, and is used to model error structures that are more general than in the regression model. Other methods such as time-series and exponential smoothing models formulate the correlation between observation values as parameters, and then estimate parameters using data and forecast response variable using estimates. When time-series and exponential smoothing models were first developed, these methods were not devised to analyze relationships between explanatory and dependent variables. Wang [50] proposed an exponential smoothing model that included explanatory variables to explore the association of research variable. Given that this method is a relatively new development, most statistical software packages have not yet incorporated the related functions, hence this method has not become widely used. By contrast, the LME model was developed more than 30 years ago, and related functions have been included in various statistical software packages. Using the models in this paper to forecast product demand may yield negative predictive values. Negative values are usually obtained from historical data where product demand is zero or very low. To prevent this from happening, the predictive value was truncated at 0 (i.e., $F_{t}=\max \left(\hat{Y}_{t}, 0\right)$, where $\hat{Y}_{t}$ denotes a predictive value derived from any method, and $F_{t}$ denotes an actual predictive value obtained from any prediction method). In other words, if $\hat{Y}_{t}>0$, then $F_{t}=\hat{Y}_{t}$; if $\hat{Y}_{t} \leq 0$, then $F_{t}=0$. Some link functions in generalized LME models can deal with cases where dependent variables are restricted to $Y_{t} \geq 0$ [35]. However, the prediction intervals for the random-effects in the LME model are well developed [17, 39, 5, 14, 53]. It is useful to applying the prediction intervals in business operations is useful in knowing whether a randomeffect exists. Implementing an MTS strategy enhances the competitive advantage of a manufacturer provides the ability to rapidly satisfy product demand, thereby reducing internal and external transaction costs in handling orders. Employing this strategy also enables high batch centralized production, reducing production costs and assisting manufacturers in negotiating material costs with suppliers. Given that this approach gives short delivery times, customer satisfaction is improved, thus attracting potential customers who need products im- mediately. Consequently, this 
leads to incrased market share. The MTS strategy also improves the usage rate of production equipment. Companies that adopt an MTS strategy require an accurate forecasting method to realize these advantages. This study has proposed an accurate forecasting method for deter- mining the stock levels a company requires when adopting the MTS strategy, a topic that has seldom been discussed in studies on the MTS strategy. Using an MTS strategy involves the potential risk of increasing inventory costs. Therefore, ther recommendation for future studies is to adequately apply the strengths of the LME model (e.g., accurately forecasting demand for multiple product types in one go) when forecasting. Future studies might consider investigating whether the forecasting intervals of the LME model can be coupled with various inventory strategies to assist manufacturers in adopting the MTS strategy in order to develop an optimal business operation model in terms of optimal inventory time points and minimal inventory costs. In addition, to remain competitive, companies should improve their organizational capability to raise the threshold at which competitors develop similar operating models. Future studies might also explore the benefits that the MTS strategy involving an LME model brings to the various departments of an enterprise and the effects of such a strategy on customer satisfaction and loyalty.

\section{References}

[1] Y. Acar and E. S Gardner (2012). Forecasting method selection in a global supply chain. International Journal of Forecasting 28(4), 842-848.

[2] I. JBF Adan and J. Van der Wal (1998). Combining make to order and make to stock. Operations-Research-Spektrum 20(2), 73-81.

[3] P. G. Allen and B. J Morzuch (2006). Twenty-five years of progress, problems, and conflicting evidence in econometric forecasting. what about the next 25 years? International Journal of Forecasting 22(3), 475-492.

[4] J. Arnerić, T. Poklepović and Z. Aljinović (2014). Garch based artificial neural networks in forecasting conditional variance of stock returns. Croatian Operational Research Review 5(2), 329-343.

[5] R. Basu, J.K. Ghosh and R. Mukerjee (2003). Empirical bayes prediction intervals in a normal regression model: higher order asymptotics. Statistics \& probability letters 63(2), 197-203.

[6] G. Bee-Hua (1999). An evaluation of the accuracy of the multiple regression approach in forecasting sectoral construction demand in singapore. Construction Management \& Economics 17(2), 231-241.

[7] V. Bianco, O. Manca and S. Nardini (2009). Electricity consumption forecasting in italy using linear regression models. Energy 34(9), 1413-1421.

[8] R. Blackburn, K. Lurz, B. Priese, R. Göb and I.-L. Darkow (2014). A predictive analytics approach for demand forecasting in the process industry. International Transactions in Operational Research.

[9] M. L Bourbonnais, T. A Nelson, M. RL Cattet, C. T Darimont, G. B Stenhouse and D. M Janz (2014). Environmental factors and habitat use influence body condition of individuals in a species at risk, the grizzly bear. Conservation Physiology 2(1), cou043.

[10] G. E Box (1976). Time series analysis: Forecasting and control. Time Series and Digital Processing. 
[11] G. EP Box and G. C Tiao (1975). Intervention analysis with applications to economic and environmental problems. Journal of the American Statistical association 70(349), 70-79.

[12] L. Breiman, et al (2001). Statistical modeling: The two cultures (with comments and a rejoinder by the author). Statistical Science 16(3), 199-231.

[13] Y. Cao, G. Wan and F. Wang (2011). Predicting financial distress of chinese listed companies using rough set theory and support vector machine. Asia-Pacific Journal of Operational Research 28(01), 95-109.

[14] S. Chatterjee, P. Lahiri and H. Li (2008). On small area prediction interval problems. The Annals of Statistics 36, 1221-1245.

[15] F. Chen, J. K Ryan and D. Simchi-Levi (2000). The impact of exponential smoothing forecasts on the bullwhip effect. Naval Research Logistics 47(4), 269-286.

[16] M.-K. Chen and A. Cheng (2003). The study of supply chain inventory strategy under bto production environment. Journal of the Chinese Institute of Industrial Engineers 20(4), 398-410.

[17] DR Cox (1975). Prediction intervals and empirical bayes confidence intervals. Perspectives in Probability and Statistics, 47-55.

[18] J. G De Gooijer and R. J Hyndman (2006). 25 years of time series forecasting. International journal of forecasting 22(3), 443-473.

[19] I. Durdević Babić (2015). Predicting student satisfaction with courses based on log data from a virtual learning environment-a neural network and classification tree model. Croatian Operational Research Review 6(1), 105-120.

[20] E Erdogdu (2007). Electricity demand analysis using cointegration and arima modelling: A case study of turkey. Energy policy 35(2), 1129-1146.

[21] R. Fildes (2006). The forecasting journals and their contribution to forecasting research: Citation analysis and expert opinion. International Journal of forecasting $22(3), 415-432$.

[22] S. Gelper, R. Fried and C. Croux (2010). Robust forecasting with exponential and holt-winters smoothing. Journal of forecasting 29(3), 285-300.

[23] K. Gilbert (2005). An arima supply chain model. Management Science 51(2), 305-310.

[24] R. Göb, K. Lurz and A. Pievatolo (2013). Electrical load forecasting by exponential smoothing with covariates. Applied Stochastic Models in Business and Industry 29(6), 629-645.

[25] S. C Graves (1999). A single-item inventory model for a nonstationary demand process. Manufacturing \& Service Operations Management 1(1), 50-61.

[26] W. M. Guillot (2003). Strategic leadership: Defining the challenge. Air and Space Power Journal 4.

[27] L. C Hamilton and D. Press (1992). Regression with graphics: A second course in applied statistics, volume 1. Duxbury Press Belmont, CA.

[28] J. Hanke (1989). Forecasting in business schools: A follow-up survey. International Journal of Forecasting 5(2), 259-262.

[29] L. C Hendry and BG Kingsman (1989). Production planning systems and their applicability to make-to-order companies. European Journal of Operational Research 40(1), 1-15.

[30] P. Hiesl and J. G Benjamin (2014). Estimating processing times of harvesters in thinning operations in maine. Forest Products Journal.

[31] T. G Holmes, W. M Tonn, C. A Paszkowski and G. J Scrimgeour (2014). Effects of winter surface aeration on pelagic zooplankton communities in a small boreal foothills lake of alberta, Canada. Journal of Freshwater Ecology, (ahead-of-print), 1-14.

[32] C. C Holt (2004). Forecasting seasonals and trends by exponentially weighted moving 
averages. International Journal of Forecasting 20(1), 5-10.

[33] W.-C. Hong, Y. Dong, L.-Y. Chen and C.-Y. Lai (2010). Taiwanese 3g mobile phone demand forecasting by svr with hybrid evolutionary algorithms. Expert Systems with Applications 37(6), 4452-4462.

[34] W.-Y. Hwang and J.-S. Lee (2014). A new forecasting scheme for evaluating long-term prediction performances in supply chain management. International Transactions in Operational Research 21(6), 1045-1060.

[35] J. Jiang (2007). Linear and generalized linear mixed models and their applications. Springer Science \& Business Media.

[36] N. M Laird and J. H Ware (1982). Random-effects models for longitudinal data. Biometrics, 963-974.

[37] R. R Magee et al (1998). Strategic leadership primer. Technical report, DTIC Document.

[38] N. Mohsin, G. Mourad, M. Faure, I. Szawarc and J. Bringer (2013). Metabolic syndrome performs better than the individual factors in predicting renal graft outcome. In Transplantation proceedings, volume 45, Elsevier, 3517-3519.

[39] C. N Morris (1983). Parametric empirical bayes inference: theory and applications. Journal of the American Statistical Association 78(381), 47-55.

[40] J. F Muth (1960). Optimal properties of exponentially weighted forecasts. Journal of the american statistical association 55(290), 299-306.

[41] P.-F. Pai, K.-P. Lin and J.-S. Wang (2004). Stock price forecasting in taiwan using ellipsoidal fuzzy system. Journal of the Chinese Institute of Industrial Engineers 21(2), 146-155.

[42] A. Pankratz (1991). Forecasting with dynamic regression models. Wiley Series in Probability and Mathematical Statistics. Applied Probability and Statistics, New York: Wiley.

[43] J. C Pinheiro and D. M Bates (2010). Mixed-effects models in S and S-PLUS. Springer Science \& Business Media.

[44] W Popp (1965). Simple and combined inventory policies, production to stock or to order? Management Science 11(9), 868-873.

[45] S. Rajagopalan (2002). Make to order or make to stock: model and application. Management Science 48(2), 241-256.

[46] A. Schwalm, Y.-S. Feng, J. Moock and T. Kohlmann (2014). Differences in eq-5d31 health state valuations among patients with musculoskeletal diseases, health care professionals and healthy volunteers. The European Journal of Health Economics, $1-13$.

[47] S. A Shambach (2004). Strategic leadership primer. Technical report, DTIC Document.

[48] C. A. Soman, D. P. Van Donk and G. Gaalman (2004). Combined make-to-order and make-to-stock in a food production system. International Journal of Production Economics 90(2), 223-235.

[49] Y.-C. Tsai, Y.-W. Chiu, J.-C. Tsai, H.-T. Kuo, S.-C. Lee, C.-C. Hung, M.-Y. Lin, S.-J. Hwang, M.-C. Kuo and H.-C. Chen (2014). Association of angiopoietin-2 with renal outcome in chronic kidney disease. PloS one 9(10), e108862.

[50] S. Wang (2006). Exponential smoothing for forecasting and Bayesian validation of computer models. PhD thesis, Georgia Institute of Technology.

[51] B. T. West, K. B. Welch and A. T. Galecki (2014). Linear mixed models: a practical guide using statistical software. CRC Press.

[52] T.M. Williams (1984). Special products and uncertainty in production/inventory systems. European Journal of Operational Research 15(1), 46-54. 
[53] M. Yoshimori, P. Lahiri, et al (2014). A second-order efficient empirical bayes confidence interval. The Annals of Statistics 42(4), 1-29. 